\title{
COMBINATORIAL CURVE NEIGHBORHOODS FOR THE AFFINE FLAG MANIFOLD OF TYPE $A_{1}^{1}$
}

\author{
LEONARDO C. MIHALCEA AND TREVOR NORTON
}

\begin{abstract}
Let $X$ be the affine flag manifold of Lie type $A_{1}^{1}$. Its moment graph encodes the torus fixed points (which are elements of the infinite dihedral group $\left.D_{\infty}\right)$ and the torus stable curves in $X$. Given a fixed point $u \in D_{\infty}$ and a degree $\mathbf{d}=\left(d_{0}, d_{1}\right) \in \mathbb{Z}_{\geq 0}^{2}$, the combinatorial curve neighborhood is the set of maximal elements in the moment graph of $X$ which can be reached from $u$ using a chain of curves of total degree $\leq \mathbf{d}$. In this paper we give a formula for these elements, using combinatorics of the affine root system of type $A_{1}^{1}$.
\end{abstract}

\section{INTRODUCTION}

Let $X$ be an arbitrary algebraic variety and $\Omega \subset X$ be a subvariety. Fix a degree d, i.e. an effective homology class in $H_{2}(X)$. The (geometric) curve neighborhood $\Gamma_{\mathbf{d}}(\Omega)$ is the locus of points $x \in X$ which can be reached from $\Omega$ by a rational curve of some effective degree $\leq \mathbf{d}$. For example, if $X=\mathbb{P}^{2}$ is the projective plane, and $\Omega=p t$, then any other point in $X$ can be reached from the given point, using a projective line. This implies that $\Gamma_{1}(p t)=\mathbb{P}^{2}$.

Curve neighborhoods have been recently defined by A. Buch and the first named author in [1], in relation to the study of quantum cohomology and quantum K theory rings of generalized flag manifolds $X=G / B$, where $G$ is a complex semisimple Lie group, and $B$ a Borel subgroup. The curve neighborhoods which are relevant in that context are those when $\Omega$ is a Schubert variety in $G / B$. It turns out that in this situation the calculation of the curve neighborhoods is encoded in the moment graph of $X$. This is a graph encoding the $T$-fixed points and the $T$-stable curves in $X$, where $T$ is a maximal torus of $G$. Similar considerations, but in the case when $X$ is an affine flag manifold, led L. Mare and the first named author to a definition of an affine version of the quantum coohmology ring; see [4]. The curve neighborhoods which were relevant for quantum cohomology calculations were those for certain "small" degrees. Those for "large" degrees, which seem to encode more refined information about the geometry and the combinatorics of affine flag manifolds, are still unknown.

In the current paper we give an explicit combinatorial formula for the curve neighborhoods of the simplest affine flag manifold, that of affine Lie type $A_{1}^{1}$. See e.g. [3] for details on affine flag manifolds. Instead of introducing the geometry related to this flag manifold we consider the more elementary - but equivalent -

Date: December 13, 2015.

2010 Mathematics Subject Classification. Primary 05E15; Secondary: 17B67, 14M15.

L. C. Mihalcea was supported in part by NSA Young Investigator Award H98230-13-1-0208 and a Simons Collaboration Grant. 
problem of calculating the combinatorial curve neighborhoods. These are encoded in the combinatorics of moment graph of the affine flag manifold.

To state our main result, we briefly introduce some notation and recall few definitions. Full details are given in section 2 below. Let $D_{\infty}$ be the infinite dihedral group, generated by reflections $s_{0}$ and $s_{1}$. (This is the affine Weyl group of Lie type $A_{1}^{1}$.) Each element of $D_{\infty}$ has a unique reduced expression which involves $a s_{0}$ 's and $b s_{1}$ 's, where $|a-b| \leq 1$. There is a natural length function $\ell: D_{\infty} \rightarrow \mathbb{Z}_{\geq 0}$, and a (Bruhat) partial order on $D_{\infty}$, denoted $<$. A degree $\mathbf{d}$ is a pair of nonnegative integers $\left(d_{0}, d_{1}\right)$. The moment graph has vertices given by the elements of $D_{\infty}$; there is an edge between $u, v \in D_{\infty}$ whenever there exists an (affine root) reflection $s_{(a, b)}$ such that $v=u s_{(a, b)}$. This edge has degree $\mathbf{d}=(a, b)$ such that $|a-b|=1$; see $\S 2.2$ below. A chain in the moment graph is a succession of adjacent edges, and it has the degree the sum of degrees of each of its edges.

Finally, fix $\mathbf{d}=\left(d_{0}, d_{1}\right)$ a degree, and $u \in D_{\infty}$. The (combinatorial) curve neighborhood $\Gamma_{\mathbf{d}}(u)$ is the set of elements in $D_{\infty}$ such that: (1) they can be joined to $u$ (in the moment graph) by a chain of degree $\leq \mathbf{d}$; and (2), they are maximal among all elements satisfying (1). To each $u \in D_{\infty}$ one associates the degree $d(u):=(a, b)$ where $u$ has a reduced expression with $a s_{0}$ 's and $b s_{1}$ 's.

Consider the set

$$
\mathcal{A}_{\mathbf{d}}(u):=\left\{v \in D_{\infty}: \ell(u v)=\ell(u)+\ell(v), d(v) \leq \mathbf{d}\right\},
$$

and denote by $\max \mathcal{A}_{\mathbf{d}}(u)$ the subset of its maximal elements. Our main result is:

Theorem 1.1. Let $u \in D_{\infty}$ and $\mathbf{d}=\left(d_{1}, d_{2}\right)$ a degree. Then the following hold:

(a) The curve neighborhood $\Gamma_{\mathbf{d}}(u)$ is given by

$$
\Gamma_{\mathbf{d}}(u)=\left\{u w: w \in \max \mathcal{A}_{\mathbf{d}}(u)\right\} .
$$

(b) Formulas (3) and (4) below give explicit combinatorial formulas for the elements in $\max \mathcal{A}_{\mathbf{d}}(u)$. In particular, the curve neighborhood $\Gamma_{\mathbf{d}}(u)$ has exactly two elements if $u=1$ and $\mathbf{d}=(a, a)$, and one element otherwise.

It is interesting to remark that the curve neighborhoods distinguish the degrees corresponding to "imaginary roots" $(a, a)$ in this case. (See [2] for more about this affine root system.) We plan to study further this phenomenon elsewhere. The theorem implies that the "geometric" curve neighborhood for the Schubert variety indexed by $u$ is either a single Schubert variety, or the union of two Schubert varieties, indexed by the elements in $\Gamma_{\mathbf{d}}(u)$. We refer to [4] for a discussion of geometric curve neighborhoods.

This paper is the outcome of an undergraduate research project of T.N. conducted under the direction of L.M.

\section{Preliminaries}

2.1. The infinite dihedral group. The infinite dihedral group $D_{\infty}$ is the group with generators $s_{0}, s_{1}$ and relations $s_{0}^{2}=s_{1}^{2}=1$. Each element $w \in D_{\infty}$ can be written uniquely as a product of $s_{0}$ 's and $s_{1}$ 's in such a way that no $s_{0}$ 's and no $s_{1}$ 's are consecutive. We call such an expression reduced. We define the length $\ell(w)$ of $w$ to be the total number of $s_{0}$ 's and $s_{1}$ 's in the expression of $w$. For example, $\ell\left(s_{0}\right)=1$ and $\ell\left(s_{1} s_{0} s_{1} s_{0}\right)=4$.

A (positive) root corresponding to $D_{\infty}$ is a pair of non-negative integers $\alpha=$ $(a, b) \in \mathbb{Z}_{\geq 0}^{2}$ such that $|a-b|=1$. For example, $\alpha_{0}:=(1,0)$ and $\alpha_{1}:=(0,1)$ are 
roots, and so is $\alpha=2 \alpha_{0}+3 \alpha_{1}=(2,3)$. Fix $\alpha=\left(a_{0}, a_{1}\right)$ a root. A root reflection $s_{\alpha}$ is the unique element of $D_{\infty}$ which can be written as a product of $a_{0} s_{0}$ 's and $a_{1} s_{1}$ 's, and which has length $a+b$. For example:

$$
s_{(2,3)}=s_{1} s_{0} s_{1} s_{0} s_{1} ; \quad s_{(1,0)}=s_{0} .
$$

The terminology follows from the fact that these are the positive roots of the affine Lie algebra of type $A_{1}^{(1)}$; see e.g. [2].

We record for later use the following properties:

Lemma 2.1. Let $u, v \in D_{\infty}$. Then:

(a) $\ell(u)=\ell\left(u^{-1}\right)$;

(b) $u$ is a root reflection if and only if $\ell(u)$ is odd;

(c) $\ell(u v) \leq \ell(u)+\ell(v)$;

(d) If $\ell(u) \leq \ell(v)$, then $\ell(u v)=\ell(u)+\ell(v)$ or $\ell(u v)=\ell(v)-\ell(u)$. In particular, $\ell(u v) \equiv \ell(u)+\ell(v) \bmod 2$.

Proof. This is an easy verification.

2.2. The moment graph and curve neighborhoods. The moment graph $G$ associated to $D_{\infty}$ is the graph given by the following data:

- The set of $V$ of vertices is the group $D_{\infty}$;

- Let $u, v \in V$ be vertices. Then there is an edge from $u$ to $v$ iff there exists a root $\alpha=\left(a_{0}, a_{1}\right)$ such that $v=u s_{\alpha}$. We denote this situation by

$$
u \stackrel{\alpha}{\longrightarrow} v
$$

and we say that the degree of this edge is $\alpha$.

In the figure below we show the moment graph up to elements of length 4 . We labeled few of the edges by their corresponding degrees.

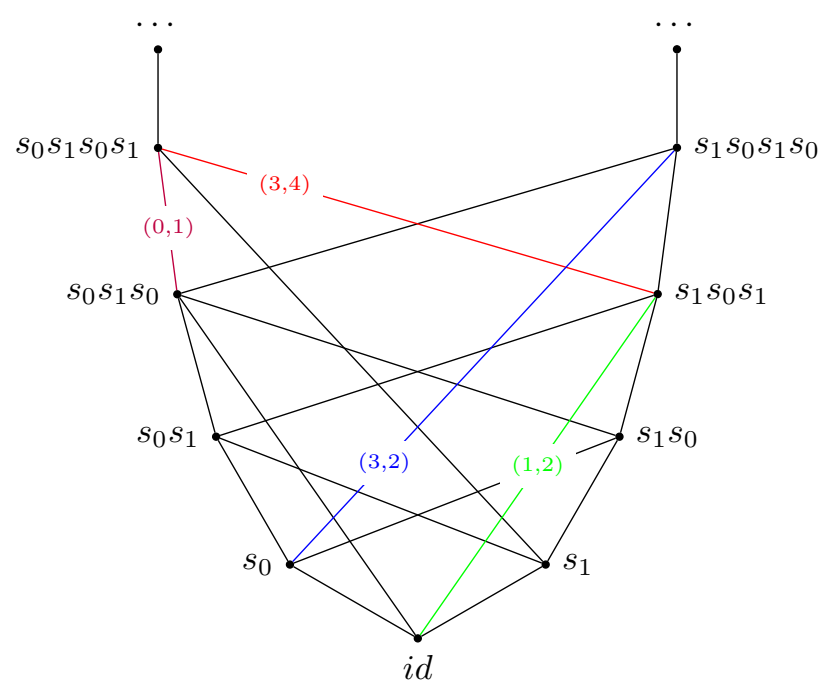

Remark 2.2. As mentioned in the introduction, the vertices of this graph correspond to the $T$-fixed points, and its edges to the $T$-stable curves in the affine flag manifold of type $A_{1}^{1}$, where $T$ is a maximal torus in an affine Kac-Moody group of type $A_{1}^{1}$. See e.g. [3, Ch. 12], especially §12.2.E for details. 
A chain between $u$ and $v$ in the moment graph is a succession of adjacent edges starting with $u$ and ending with $v$

$$
\pi: u=u_{0} \stackrel{\beta_{0}}{\longrightarrow} u_{1} \stackrel{\beta 1}{\longrightarrow} \ldots \stackrel{\beta_{n-2}}{\longrightarrow} u_{n-1} \stackrel{\beta_{n-1}}{\longrightarrow} u_{n}=v .
$$

The chain is called increasing if at each step the lengths increase, i.e. $\ell\left(u_{i}\right)>\ell\left(u_{i-1}\right)$ for $1 \leq i \leq n$. The degree of the chain $\pi$ is $\operatorname{deg}(\pi)=\beta_{0}+\cdots+\beta_{n-1}$. Define a partial ordering on the elements of $D_{\infty}$ by $u<v$ iff there exists an increasing chain starting with $u$ and ending with $v$.

The next result gives an equivalent way to describe the partial ordering on $D_{\infty}$ :

Lemma 2.3. Let $u, v \in D_{\infty}$. Then $u<v$ if and only if $\ell(u)<\ell(v)$.

Proof. Clearly if $u<v$ then $\ell(u)<\ell(v)$ from the definition of an increasing chain. To prove the converse, we first notice that if $\ell(v)-\ell(u)=1$, then $u^{-1} v$ is a root reflection $s_{\alpha}$ (possibly of length $>1$ ), thus there exists an edge $u \stackrel{\alpha}{\longrightarrow} v$. The general statement follows by induction on $\ell(v)-\ell(u) \geq 1$.

A degree is a pair of nonnegative integers $\mathbf{d}=\left(d_{0}, d_{1}\right)$. There is a natural partial order on degrees. If $\mathbf{d}=\left(d_{0}, d_{1}\right)$ and $\mathbf{d}^{\prime}=\left(d_{0}^{\prime}, d_{1}^{\prime}\right)$ then $\mathbf{d} \geq \mathbf{d}^{\prime}$ iff $d_{i} \geq d_{i}^{\prime}$ for $i \in\{0,1\}$.

Definition 2.4. Fix $\mathbf{d}$ a degree and $u \in D_{\infty}$. The (combinatorial) curve neighborhood is the set $\Gamma_{\mathbf{d}}(u)$ consisting of elements $v \in D_{\infty}$ such that:

(1) there exists a chain of some degree $\mathbf{d}^{\prime} \leq \mathbf{d}$ from $u$ to $v$ in the moment graph $G$;

(2) The elements $v$ are maximal among all of those satisfying the condition in (1).

For example:

$$
\Gamma_{(1,0)}(i d)=\Gamma_{(2,0)}(i d)=\left\{s_{0}\right\} ; \quad \Gamma_{(1,1)}(i d)=\left\{s_{1} s_{0}, s_{0} s_{1}\right\} .
$$

The main question of the project is to find a formula to determine $\Gamma_{\mathbf{d}}(u)$.

For $w \in D_{\infty}$ define the degree associated to $w$ to be $d(w)=\left(d_{0}, d_{1}\right)$ where $d_{i}:=$ number of reflections $s_{i}$ in the reduced word of $w$. The following holds.

Lemma 2.5. Let $u, v \in D_{\infty}$ and assume there is a chain from $u$ to $v$ of degree $\mathbf{d}$. Then $\mathbf{d}=d\left(u^{-1} v\right)+2(r, s)$ where $r, s \in \mathbb{Z}_{\geq 0}$. In particular, $\mathbf{d} \geq d\left(u^{-1} v\right)$.

Proof. Let $\beta_{0}:=\left(a_{0}, b_{0}\right), \beta_{1}:=\left(a_{1}, b_{1}\right), \ldots, \beta_{n-1}:=\left(a_{n-1}, b_{n-1}\right)$ be the labels of the edges of the chain $\pi$. Then $v=u s_{\beta_{0}} \cdots s_{\beta_{n-1}}$ and $\mathbf{d}=\beta_{0}+\cdots+\beta_{n-1}=$ $\left(a_{0}+\cdots+a_{n-1}, b_{0}+\cdots+b_{n-1}\right)$. Now $d\left(u^{-1} v\right)=d\left(s_{\beta_{0}} \cdots s_{\beta_{n-1}}\right)$. If $s_{\beta_{0}} \cdots s_{\beta_{n-1}}$ is non-reduced, one needs to perform some cancellations of the form $s_{0}^{2}=1$ or $s_{1}^{2}=1$. Each of these result in a decrease by 2 of the number of $s_{0}$ 's, respectively $s_{1}$ 's in an expression for $s_{\beta_{0}} \cdots s_{\beta_{n-1}}$. Thus $d\left(u^{-1} v\right)=\mathbf{d}-2(r, s)$ as claimed.

\section{Calculation of the Curve neighborhoods}

Let $\mathbf{d}=\left(d_{1}, d_{2}\right)$ be a degree. such that $d_{1} \neq d_{2}$. We denote by $\alpha(\mathbf{d})$ the maximal root $\alpha$ such that $\alpha \leq \mathbf{d}$. Clearly there is exactly one such root, and it is easy to find the following explicit formula for it:

$$
\alpha(\mathbf{d})= \begin{cases}\left(d_{1}, d_{1}+1\right) & \text { if } d_{1}<d_{2} \\ \left(d_{2}+1, d_{2}\right) & \text { if } d_{1}>d_{2} .\end{cases}
$$


In order to find the curve neighborhoods of an element $u \in D_{\infty}$, we need the following key result.

Lemma 3.1. Let $u \in D_{\infty}$ and $\mathbf{d}=\left(d_{1}, d_{2}\right)$ a degree. Consider the set

$$
\mathcal{A}_{\mathbf{d}}(u):=\left\{v \in D_{\infty}: \ell(u v)=\ell(u)+\ell(v), d(v) \leq \mathbf{d}\right\} .
$$

Then the following hold:

- $\mathcal{A}_{\mathbf{d}}(u)$ has a unique maximal element if $u \neq 1$ or if $u=1$ and $\mathbf{d} \neq(a, a)$ for some nonnegative integer a.

- If $\mathbf{d}=(a, a)$ and $u=1$ then the maximal elements of $\mathcal{A}_{\mathbf{d}}(u)$ are $\left(s_{0} s_{1}\right)^{a}$ and $\left(s_{1} s_{0}\right)^{a}$.

Proof. Clearly, $1 \in \mathcal{A}_{\mathbf{d}}(u)$ so $\mathcal{A}_{\mathbf{d}}(u) \neq \emptyset$. For any $v \in \mathcal{A}_{\mathbf{d}}(u), d(v) \leq \mathbf{d}$, hence the set $\mathcal{A}_{\mathbf{d}}(u)$ is finite. Then it must contain a maximal element. Lemma 2.3 implies that there can be at most two maximal elements $v_{1}$ and $v_{2}$ and they must have the same length. We consider each of the situations in the statement:

Case 1. $u \neq 1$. Assume there are two maximal elements $v_{1}, v_{2}$. Since $u \neq 1$ either $u v_{1}$ or $u v_{2}$ is not reduced, say $u v_{1}$. Then $\ell\left(u v_{1}\right)<\ell(u)+\ell\left(v_{1}\right)$, and this contradicts that $v_{1} \in \mathcal{A}_{\mathbf{d}}(u)$.

Case 2. $u=1$. In this case the set $\mathcal{A}_{\mathbf{d}}(u)$ coincides with the set of all $v \in D_{\infty}$ such that $d(v) \leq \mathbf{d}$. From the description of $D_{\infty}$, it follows that $d(v)=(a, a)$ or $d(v)=(a, a+1)$ or $d(v)=(a+1, a)$ for some non-negative integer $a$. Further, the reduced decomposition of $v$ is is known in each case: there are two possibilities for $v$ if $d(v)=(a, a)$, and there is exactly one (in fact $\left.v=s_{\alpha(\mathbf{d})}\right)$ in the other two. The claim follows from this.

In what follows we will denote by $\max \mathcal{A}_{\mathbf{d}}(u)$ the set of maximal elements in the (finite) partially ordered set $\mathcal{A}_{\mathbf{d}}(u)$. Our main result is:

Theorem 3.2. Let $u \in D_{\infty}$ and $\mathbf{d}=\left(d_{1}, d_{2}\right)$ a degree. Then

$$
\Gamma_{\mathbf{d}}(u)=\left\{u w: w \in \max \mathcal{A}_{\mathbf{d}}(u)\right\} .
$$

We will prove this theorem in the next two sections, which correspond to the cases $u=1$ and $u \neq 1$. For now, notice that the proof of Lemma 3.1, and some easy arguments based on reduced decompositions in $D_{\infty}$, imply that if $u=1$ then the set of maximal elements of $\mathcal{A}_{\mathbf{d}}(1)$ is the following:

$$
\max \mathcal{A}_{\mathbf{d}}(1)= \begin{cases}\left\{s_{\alpha(\mathbf{d})}\right\} & \text { if } \mathbf{d}=\left(d_{1}, d_{2}\right) \text { and } d_{1} \neq d_{2} ; \\ \left\{\left(s_{0} s_{1}\right)^{a},\left(s_{1} s_{0}\right)^{a}\right\} & \text { if } \mathbf{d}=(a, a) .\end{cases}
$$

If $u \neq 1$, we assume for simplicity that that last simple reflection in the reduced word for $u$ is $s_{0}$, i.e. $u=\cdots s_{0}$. (The other situation will be symmetric). Then

$$
\max \mathcal{A}_{\mathbf{d}}(u)= \begin{cases}\left\{s_{1} s_{\alpha(\mathbf{d}-(0,1))}\right\} & \text { if } d_{0}=d_{1} \\ \left\{s_{\alpha(\mathbf{d})}\right\} & \text { if } d_{1}>d_{0} \\ \left\{s_{0} s_{\alpha(\mathbf{d})}\right\} & \text { if } d_{1}<d_{0}\end{cases}
$$

The two formulas give explicit combinatorial rules to determine the curve neighborhood $\Gamma_{\mathbf{d}}(u)$. See section 3.3 below for several examples. 


\subsection{Curve neighborhoods for $u=1$.}

Theorem 3.3. Let $\mathbf{d}=\left(d_{1}, d_{2}\right)$ be a degree. Then the curve neighborhood of the identity can be calculated in the following way:

$$
\Gamma_{\mathbf{d}}(1)=\max \mathcal{A}_{\mathbf{d}}(1)= \begin{cases}\left\{s_{\alpha(\mathbf{d})}\right\} & \text { if } d_{1} \neq d_{2} ; \\ \left\{\left(s_{0} s_{1}\right)^{a},\left(s_{1} s_{0}\right)^{a}\right\} & \text { if } \mathbf{d}=(a, a) .\end{cases}
$$

Proof. If $v \in \Gamma_{\mathbf{d}}(1)$ then there exists a chain of degree $\leq \mathbf{d}$ joining 1 to $v$. Then by Lemma $2.5 \mathbf{d} \geq d(v)$. In particular $v \in \mathcal{A}_{\mathbf{d}}(1)$, thus $\Gamma_{\mathbf{d}}(1) \subset \mathcal{A}_{\mathbf{d}}(1)$, and the inclusion is compatible with the partial order $<$. Conversely, if $v$ is any element in $\mathcal{A}_{\mathbf{d}}(1)$ then there exists a chain of degree $d(v) \leq \mathbf{d}$ joining 1 to $v$. If $v$ is maximal in $\mathcal{A}_{\mathbf{d}}(1)$, and because $\Gamma_{\mathbf{d}}(1) \subset \mathcal{A}_{\mathbf{d}}(1)$, it follows that $v \in \Gamma_{\mathbf{d}}(1)$. This finishes the proof.

3.2. General curve neighborhoods. The goal of this section is to find a formula for the curve neighborhoods $\Gamma_{d}(u)$ for $u \neq 1$ and $\mathbf{d} \neq(0,0)$. First we need some preparatory lemmas.

Lemma 3.4. Let $u \in D_{\infty}, z \in \mathcal{A}_{\mathbf{d}}(1)$ and $v \in \Gamma_{\mathbf{d}}(u)$. Then:

(a) $\ell(u z) \leq \ell(v)$ and $d\left(u^{-1} v\right) \leq \mathbf{d}$.

(b) Let $z \in \Gamma_{\mathbf{d}}(1)$ (i.e. $z$ is maximal in $\mathcal{A}_{\mathbf{d}}(1)$ ). Then $\ell\left(u^{-1} v\right) \leq \ell(z)$.

Proof. Since $z \in \mathcal{A}_{\mathbf{d}}(1)$ there exists a chain of degree $d(z) \leq \mathbf{d}$ joining 1 to $z$. Multiplying this chain by $u$ on the left gives a chain between $u$ and $u z$ of the same degree. The first statement in (a) follows by maximality of $v$. To prove the second statement in (a), notice that since $v \in \Gamma_{\mathbf{d}}(u)$ there exists a chain from $u$ to $v$ of degree $\leq \mathbf{d}$. If we multiply each element of this chain on the left by $u^{-1}$ we obtain a chain from 1 to $u^{-1} v$ of the same degree. The fact that $d\left(u^{-1} v\right) \leq \mathbf{d}$ follows from Lemma 2.5. Finally, the statement from (b) follows from the maximality of $z$, using also that maximal elements in $\mathcal{A}_{\mathbf{d}}(1)$ have the same length, by (3).

The following lemma gives a strong constraint on the possible elements in $\Gamma_{\mathbf{d}}(u)$.

Lemma 3.5. Let $v \in \Gamma_{\mathbf{d}}(u)$. Then $u^{-1} v \in \mathcal{A}_{\mathbf{d}}(u)$.

Proof. We have seen in Lemma 3.4 that $d\left(u^{-1} v\right) \leq \mathbf{d}$. It remains to show that $\ell(v)=\ell(u)+\ell\left(u^{-1} v\right)$. This clearly holds for $u=1$ and from now on we assume that $u \neq 1$. From Lemma 2.1(c) it follows that $\ell(v)=\ell\left(u u^{-1} v\right) \leq \ell(u)+\ell\left(u^{-1} v\right)$. If the inequality is strict then $\ell\left(u^{-1} v\right)>\ell(v)-\ell(u)=\ell(v)-\ell\left(u^{-1}\right)$. But $\ell(u) \leq \ell(v)$, thus by Lemma 2.1(d) it follows that

$$
\ell\left(u^{-1} v\right)=\ell(u)+\ell(v) .
$$

Consider now an element $z \in \Gamma_{\mathbf{d}}(1)=\max \mathcal{A}_{\mathbf{d}}(1)$ (by Theorem 3.3). We invoke Lemma 3.4 to obtain that

$$
\ell(u z) \leq \ell(v)<\ell(u)+\ell\left(u^{-1} v\right) \leq \ell(u)+\ell(z) .
$$

This implies that the expression $u z$ is not reduced. But since $u \neq 1$ we can eliminate the first simple reflection from the reduced expression for $z$ to define $z^{\prime}<z$ such that $\ell\left(z^{\prime}\right)=\ell(z)-1$ and $\ell\left(u z^{\prime}\right)=\ell(u)+\ell\left(z^{\prime}\right)$. Notice that $d\left(z^{\prime}\right)<d(z) \leq \mathbf{d}$ thus $z^{\prime} \in \mathcal{A}_{\mathbf{d}}(1)$. Then we have the following inequalities:

$$
\ell(v) \geq \ell\left(u z^{\prime}\right)=\ell(u)+\ell(z)-1 \geq \ell(u)+\ell\left(u^{-1} v\right)-1=\ell(u)+\ell(u)+\ell(v)-1,
$$


where the first inequality follows from Lemma 3.4(a) and the last inequality from Lemma 3.4(b). Taking the extreme sides and substracting $\ell(v)$ we obtain that $0 \geq 2 \ell(u)-1$, which is impossible since $\ell(u) \geq 1$. Thus $\ell(v)=\ell(u)+\ell\left(u^{-1} v\right)$ and this finishes the proof.

We are ready to prove our main result.

Theorem 3.6. Let $\mathbf{d}=\left(d_{1}, d_{2}\right)$ be a non-zero degree and $u \in D_{\infty}$. Then

$$
\Gamma_{\mathbf{d}}(u)=\left\{u w: w \in \max \mathcal{A}_{\mathbf{d}}(u)\right\} .
$$

Proof. Let $v \in \Gamma_{\mathbf{d}}(u)$. Then Lemma 3.5 implies that $u^{-1} v \in \mathcal{A}_{\mathbf{d}}(u)$. From Lemma 3.1 (or equation (4)) there exists a unique maximal element of $\mathcal{A}_{\mathbf{d}}(u)$, call it $w$. Then $u^{-1} v \leq w$ and clearly $w$ is also in $\mathcal{A}_{\mathbf{d}}(1)$. By Lemma 3.4(a), we deduce that $\ell(u w) \leq \ell(v)$. Then

$$
\ell(u)+\ell\left(u^{-1} v\right)=\ell(v) \geq \ell(u w)=\ell(u)+\ell(w) .
$$

This implies that $\ell\left(u^{-1} v\right) \geq \ell(w)$. Together with $u^{-1} v \leq w$, this forces $u^{-1} v=w$, i.e. $v=u w$ as claimed.

3.3. Examples. We provide several examples determining $\Gamma_{\mathbf{d}}(u)$.

- Let $u=1$ and $\mathbf{d}=(9,4)$. From (1) we obtain $\alpha(\mathbf{d})=(5,4)$, thus

$$
\Gamma_{(9,4)}(1)=\left\{s_{(5,4)}\right\}=\left\{s_{0} s_{1} s_{0} s_{1} s_{0} s_{1} s_{0} s_{1} s_{0}\right\} .
$$

- Let $u=1$ and $\mathbf{d}=(4,4)$. By (3) the two maximal elements in $\mathcal{A}_{(4,4)}(1)$ are $s_{0} s_{1} s_{0} s_{1} s_{0} s_{1} s_{0} s_{1}$ and $s_{1} s_{0} s_{1} s_{0} s_{1} s_{0} s_{1} s_{0}$. Then

$$
\Gamma_{(4,4)}(i d)=\left\{s_{0} s_{1} s_{0} s_{1} s_{0} s_{1} s_{0} s_{1}, s_{1} s_{0} s_{1} s_{0} s_{1} s_{0} s_{1} s_{0}\right\} .
$$

- Let $u=s_{0} s_{1} s_{0}$ and $\mathbf{d}=(3,3)$. From (4),

$$
\max \mathcal{A}_{(3,3)}(u)=\left\{s_{1} s_{\alpha((3,3)-(0,1))}\right\}=\left\{s_{1} s_{0} s_{1} s_{0} s_{1} s_{0}\right\} .
$$

Thus $\Gamma_{(3,3)}\left(s_{0} s_{1} s_{0}\right)=\left\{\left(s_{0} s_{1} s_{0}\right)\left(s_{1} s_{0} s_{1} s_{0} s_{1} s_{0}\right)\right\}$.

- Let $u=s_{1} s_{0} s_{1}$ and $\mathbf{d}=(3,3)$. From the symmetric version of (4),

$$
\max \mathcal{A}_{(3,3)}(u)=\left\{s_{0} s_{\alpha((3,3)-(1,0))}\right\}=\left\{s_{0} s_{1} s_{0} s_{1} s_{0} s_{1}\right\} .
$$

Thus $\Gamma_{(3,3)}\left(s_{1} s_{0} s_{1}\right)=\left\{\left(s_{1} s_{0} s_{1}\right)\left(s_{0} s_{1} s_{0} s_{1} s_{0} s_{1}\right)\right\}$.

- Let $u=s_{0} s_{1} s_{0}$ and $\mathbf{d}=(9,4)$. Then $\alpha((9,4))=(5,4)$ and using (4) again $\max \mathcal{A}_{\mathbf{d}}(u)=\left\{s_{1} s_{0} s_{1} s_{0} s_{1} s_{0} s_{1} s_{0}\right\}$. Then

$$
\Gamma_{(9,4)}\left(s_{0} s_{1} s_{0}\right)=\left\{\left(s_{0} s_{1} s_{0}\right)\left(s_{1} s_{0} s_{1} s_{0} s_{1} s_{0} s_{1} s_{0}\right)\right\} .
$$

- Let $u=s_{0} s_{1} s_{0}$ and $d=(4,9)$. Then $\alpha((4,9))=(4,5)$ and $\max \mathcal{A}_{\mathbf{d}}(u)=$ $\left\{s_{1} s_{0} s_{1} s_{0} s_{1} s_{0} s_{1} s_{0} s_{1}\right\}$. From this we obtain that

$$
\Gamma_{(9,4)}\left(s_{0} s_{1} s_{0}\right)=\left\{\left(s_{0} s_{1} s_{0}\right)\left(s_{1} s_{0} s_{1} s_{0} s_{1} s_{0} s_{1} s_{0} s_{1}\right)\right\} .
$$

\section{REFERENCES}

[1] A. Buch and L. C. Mihalcea, Curve neighborhoods of Schubert varieties, J. Differential Geom. 99 (2015), no.2, 255 - 283.

[2] V. Kac, Infinite Dimensional Lie Algebras, Second edition, Cambridge University Press, Cambridge, 1985.

[3] S. Kumar, Kac-Moody Groups, their Flag Varieties, and Representation Theory, Progress in Mathematics, vol. 204, Birkhäuser Boston, Boston, MA, 2002.

[4] L. Mare and L. C. Mihalcea, An affine quantum cohomology ring for flag manifolds and the periodic Toda lattice, available on ar $\chi \mathrm{iv}: 1409.3587$. 
Department of Mathematics, Virginia Tech University, Blacksburg, VA 24061

E-mail address: lmihalce@math.vt.edu

E-mail address: norton15@vt.edu 